\title{
The relation between the birth process and the condition of the newborn piglet and calf
}

\author{
M. A. M. Taverne
}

Published online: 8 August 2008

(C) The Author(s) 2008

\begin{abstract}
This presentation will focus on the assessment of the condition of the piglet and calf during and shortly after birth and will review some of the physiological and clinical factors that may have played a role during their delivery and influenced their viability at birth.
\end{abstract}

Keywords Newborn calves $\cdot$ Newborn piglets

\section{Introduction}

Parturition can be defined as the physiological process by which, after a species-specific duration of pregnancy, the conceptus is transported to the outside world in such a way that both the mother and offspring will survive. Parturition forms a crucial step in animal production, because more deaths occur at or near birth than during the subsequent stages of the animals' development (Randall 1978; Silver 1992). In addition, events experienced by the dam and/or her offspring during the birth process may have an impact on their subsequent performance. The following two examples clearly illustrate this latter statement: A higher morbidity and reduced survival rate has been reported for asphyxiated liveborn piglets (Herpin et al. 1996); in cattle the increased incidence of retained placenta (after abnormal calving) results in prolonged calving intervals (van Werven et al. 1992).

The relatively low level activity of the myometrium during pregnancy already affects several physiological functions of the foetus, such as breathing movements, electrocortical activity and body movements. Yet, the regular and more powerful uterine contractions during parturition cause even more pronounced, temporary reductions in uterine blood flow, and these are associated with temporary decreases in foetal blood $\mathrm{pO}_{2}$ (Jansen et al. 1979).

M. A. M. Taverne $(\bowtie)$

Department of Farm Animal Health, Foetal and Perinatal Biology Section,

Faculty of Veterinary Medicine, Utrecht University, Marburglaan 2, $3584 \mathrm{CN}$ Utrecht, The Netherlands e-mail: m.a.m.taverne@uu.nl 
The intervals between uterine contractions during parturition are therefore crucial for restoration of uteroplacental perfusion and maintenance of foetal oxygenation. The cumulative effect of labour contractions may become apparent when the duration of the delivery process is abnormally prolonged in cases of dystocia. In addition, due to compression, traction or even rupture of the umbilical cord during the birth process and/or a (partial) dislodgement of the foetal placenta, gas transport towards and from the foetus may be impeded or even completely interrupted.

While foetal viability and oxygenation are not continuously registered or monitored during parturition in domestic animals, the farmer and/or veterinary surgeon should be able to discriminate newborn animals that require special (intensive) care or treatment to improve their chances for postnatal survival.

This presentation will focus on the assessment of the condition of the piglet and calf during and shortly after birth and will review some of the physiological and clinical factors that may have played a role during their delivery and influenced their viability at birth.

\section{The piglet during and immediately after birth}

Onset of regular uterine labour contractions in pigs takes place between 4 and 9 hours before the birth of the first piglet. During the following hours contractions are propagated along the uterine horns, both in a tubocervical and cervicotubal direction, and this causes rupturing of the terminal ends of the allantochorionic membranes between adjacent foetuses (Taverne et al. 1979a, b). As a result, the two uterine horns become continuous, slippery tubes wherein the umbilical cord keeps the displacement of piglets by uterine contractions within certain limits. When a piglet finally reaches the uterine body and enters the pelvic canal, its expulsion is supported by abdominal straining efforts of the sow. Blood sampling from chronically catheterized foetuses during birth has shown that foetal arterial $\mathrm{pH}$ and $\mathrm{pO}_{2}$ are only decreased during the final minutes before spontaneous normal births (Randall 1982). Yet, behind or near a farrowing sow different categories of newborn piglets can be encountered (van der Lende and van Rens 2003; data from Large White x Meishan crossbred F2 gilts): 1) mummified piglets of different sizes (on average 0.40 per litter) and 2) non-fresh stillborn piglets (on average 0.30 per litter), together representing all foetal losses; 3) fresh stillborn ( 0.75 per litter) and 4 ) liveborn piglets (on average 10.5 per litter). Because farrowings are not continuously observed under farm conditions, the latter two groups may include asphyxiated piglets (which either died or survived) and piglets crushed by the sow. In these cases only a thorough post mortem investigation can elucidate to which category a piglet belongs. Yet, autopsy of dead piglets is not routinely performed under practical circumstances.

Close observation of (prostaglandin-induced) farrowings, combined with a strict intervention protocol, resulted in a substantial reduction of perinatal losses and increased numbers of weaned piglets (Holyoake et al. 1995), but the additional costs of these measures have to be taken into account. The percentage of stillborn piglets normally ranges between 4 and $8 \%$ and is influenced by a large numbers of factors, such as: litter size, gestation length, duration of farrowing, piglet birth intervals, birth weight, position in the birth order (rank), presentation during delivery (anterior or posterior) and the condition (broken or intact) of the umbilical cord (Zaleski and Hacker 1993; Herpin et al. 1996; Leenhouwers et al. 1999; van Dijk et al. 2005; Canario et al. 2006). In several recent studies attempts were made to reduce stillbirth rate by speeding up the delivery process with oxytocin injections immediately after the birth of the first piglet. It was shown that, despite a significant shortening of the expulsive stage, this treatment even increased 
stillbirth rates, the number of newborn piglets with a ruptured cord, the number of piglets with meconium staining on the skin, and the need for obstetrical intervention (Mota-Rojas et al. 2002; Alonso-Spilsbury et al. 2004). So the use of oxytocin in cases of uncomplicated farrowings should be avoided.

Acid-base values in cord blood, viability scores and postnatal vitality of liveborn piglets vary widely (Herpin et al. 1996; van Dijk et al. 2006). The degree of acidosis at birth increased when piglets had a later position in the birth order; it was also higher in piglets born with posterior presentation and was associated with elevated levels of plasma catecholamines. The degree of asphyxia was negatively correlated with a neonatal viability score (based on heart rate, respiration, skin colour and attempts to stand before $1 \mathrm{~min}$ after birth). Moreover, asphyxia at birth (mixed cord blood $\mathrm{pH}<7.1$ ) was associated with reduced postnatal vitality (delayed first contact with the udder), a lower body temperature at $24 \mathrm{~h}$, and a reduced growth rate and survival at 10 days (Herpin et al. 1996). These data clearly demonstrate that the birth process has a great impact on both perinatal mortality and on postnatal morbidity and survival rate. In the pathophysiology of the late, postnatal effects of asphyxia experienced during birth, formation of free radicals during the post-ischemic reperfusion period of organs (brain, intestines) plays a crucial role in tissue damage (Peeters et al. 2002). Both in human and in animal perinatology, prophylactic treatment with compounds that inhibit the formation of these radicals may be an attractive option to prevent tissue damage caused by birth asphyxia.

\section{The calf during and immediately after birth}

Perinatal losses are also substantial in cows. We calculated some 10 years ago that out of the 2 million calves that were born in The Netherlands in 1996, some 7\% were dead at birth or died within 24 hours after birth. Of the surviving calves another $4 \%$ died during the first year of their life. The (still) substantial calf mortality not only represents an enormous economic loss, but also a welfare/ethical problem. There are data to indicate (Kindahl et al. 2002; Mee, Gustafsson, Szenci, personal communications) that stillbirth rates in dairy cattle around the world are increasing, especially in heifers. In addition, fewer of these losses presently appear to be associated with dystocia. So, for an increasing amount of these perinatal losses there is no obvious explanation.

Direct access to the calf during birth is possible once the cervix has dilated and the foetal membranes have broken. Blood gas values and $\mathrm{pH}$ in samples taken from the umbilical cord (Schuijt 1992) or the dorsal digital vein of a front leg (Szenci et al. 1988) during calving confirmed earlier data from experiments with chronically catheterized foetal calves (Comline et al. 1974) that no significant changes take place in the oxygenation and acid base balance during the most part of an uncomplicated birth process. It is only during the last hour before expulsion that a more sustained hypoxia and acidosis develops, most probably as the result of a more permanent reduction in uterine blood flow. The latter is caused by hyperstimulation of uterine contractions, because plasma oxytocin reaches peak levels at this late stage of delivery (Hydbring et al. 1999), and by involution of the now unoccupied distal part of the uterine horn. A recent study, using ultrasonic transit-time measurements of umbilical blood flow during second stage labour, demonstrated that in calves born with a jugular blood $\mathrm{pH}<7.2$, umbilical blood flow significantly decreases at the end of the expulsive stage; blood flow in the umbilical arteries and vein even ceased completely during passage of the calf's head through the vulva (Bleul et al. 2007). This corresponds rather well with earlier observations by our own group that in acidotic calves the baseline foetal heart rate significantly increases 
during the final hour before birth and that jugular plasma catecholamine levels in such calves are significantly higher at birth than in nonacidotic newborns (Jonker et al. 1996). During hypoxia, cardiac output of the calf is redistributed in favour of vital organs, like the brain, heart and adrenals, at the expense of peripheral organs like the carcass and intestinal tract (Hansson 1988). The increasing amounts of lactate and pyruvate, which are produced under hypoxic conditions, will enter the circulation when blood flow through these organs is normalized after the onset of breathing. This implies that the level of acidosis might be diagnosed as even more pronounced (and of a mixed respiratory-metabolic type) when samples are taken again several minutes after birth (Chan et al. 1993). There is ample evidence that the acid base balance and plasma catecholamine levels of newborn calves are influenced by type of delivery. Calves delivered by caesarean section clearly showed more favourable values than those delivered by forced extraction (Chan et al. 1993).

Meconium staining of the skin has only been investigated in caesarean delivered newborn calves and occurred in 9\% (Szenci et al. 1989) and 16\% of cases (Schuijt 1992). The latter author concluded that, although its occurrence was related to a nonvital (weak or stillborn) condition at birth and increased neonatal death within 7 days, meconium staining had no value for prediction of calf survival.

Viability scoring of newborn calves on the basis of criteria such as muscle tone, movements, respiration, reflex irritability and colour of mucous membranes, has been explored by many investigators (for review see Schuijt and Taverne 1994). However, these systems show very little conformity and the validity of these different methods has not been analysed. In the obstetrical practice of the Utrecht veterinary faculty, the time taken by newborn calves to attain Sternal Recumbency (T-SR) appeared to be a valuable, practicable and objective diagnostic tool to estimate their condition during the first 15 postnatal minutes (Schuijt and Taverne 1994). This parameter was evaluated in a group of 219 calves born (on the farm or at the clinic) after either unassisted parturition, caesarean section, normal extraction or a powerful/forced extraction. Calves were defined as vital if they only received routine care without medical treatment and survived during the first week of life without symptoms of illness $(n=192)$. The remaining 27 calves were designated as nonvital. Jugular blood sampling took place at 10, 20 and 30 minutes and was continued at least until $\mathrm{pH}$ was $>7.2$ and base-excess was $>-5.0 \mathrm{mmol} / \mathrm{l}$. The mean $( \pm \mathrm{SD}) \mathrm{T}-\mathrm{SR}$ value of the vital calves delivered after power/forced extraction was $9.0( \pm 3.3)$ minutes and was significantly higher than in the vital calves from the other delivery groups $(4.0 \pm 2.2,4.5 \pm$ 3.1 and $5.4 \pm 3.3 \mathrm{~min}$ for calves from the spontaneous, caesarean section and normal extraction groups, respectively). The mean T-SR value for the 27 nonvital calves was $20 \pm$ 6.6 minutes. A T-SR value of $>15$ minutes had a predictive value of $84 \%$ for non-vitality, while a jugular $\mathrm{pH}$ value at 10 minutes of $<6.9$ had a predictive value of only $68 \%$. Because the postnatal rotation of the calf to sternal recumbency represents a primary activity and reflects the functioning of the circulation, organs of locomotion and the nervous system, this component of the calf's behaviour during the immediate postnatal period deserves further study under more controlled conditions.

Open Access This article is distributed under the terms of the Creative Commons Attribution Noncommercial License which permits any noncommercial use, distribution, and reproduction in any medium, provided the original author(s) and source are credited. 


\section{References}

Alonso-Spilsbury M., Mota-Rojas D., Martinez-Brunes J., Arch E., Mayagoitia A.L., Ramirez-Necoechea R., Olmos A., Trujillo M.E., 2004. Use of oxytocin in penned sows and its effect on fetal intra-partum asphyxia. Animal Reproduction Science, 84, 157-167.

Bleul U., Lejeune B., Schwantag S., Kähn W., 2007. Ultrasonic transit-time measurement of blood flow in the umbilical arteries and veins in the bovine fetus during stage II of labor. Theriogenology, 67, 11231133 .

Canario L., Cantoni E., Le Bihan E., Caritez J.C., Billon Y., Bidanel J.P., Fouley J.L., 2006. Between-breed variability of stillbirth and its relationship with sow and piglet characteristics. Journal of Animal Science, 84, 3185-3196.

Chan W.W., Jonker F.H., Rausch W.D., Taverne M.A.M., 1993. Plasma catecholamines and blood chemistry in newborn calves in relation to different obstetrical procedures and to neonatal outcome. Animal Reproduction Science, 34, 43-54.

Comline R.S., Hall L.W., Lavelle R.B., Nathanielsz P.W., Silver M., 1974. Parturition in the cow: endocrine changes in animals with chronically implanted catheters in the foetal and maternal circulations. Journal of Endocrinology, 63, 451-472.

Hansson M.A., 1988. The importance of baro- and chemoreflexes in the control of the foetal cardiovascular system. Journal of Developmental Physiology, 10, 491-511.

Herpin P., Le Dividich J., Hulin J.C., Filaut M., De Marco F., Bertin R., 1996. Effects of the level of asphyxia during delivery on viability at birth and early postnatal vitality of newborn pigs. Journal of Animal Science, 74, 2067-2075.

Holyoake P.K., Dial G.D., Trigg T., King V.L., 1995. Reducing pig mortality through supervision during the perinatal period. Journal of Animal Science, 73, 3543-3551.

Hydbring E., Madej A., MacDonald E., Drugge-Boholm G., Berglund B., Olsson K., 1999. Hormonal changes during parturition in heifers and goats are related to phases and severity of labour. Journal of Endocrinology, 160, 75-85.

Jansen C.A.M., Krane E.J., Thomas A.L., Beck N.F.G., Lowe K.C., Joyce P., Parr M., Nathanielsz P.W., 1979. Continuous variability of foetal PO2 in the chronically catheterised foetal sheep. American Journal of Obstetrics and Gynecology, 134, 776-783.

Jonker F.H., van Geijn H.A., Chan W.W., Rausch W.D., van der Weijden G.C., Taverne M.A.M., 1996. Characteristics of fetal heart rate changes during the expulsive stage of bovine parturition in relation to fetal outcome. American Journal of Veterinary Research, 57, 1373-1381.

Kindahl H., Kornmatisuk B., Königsson K., Gustafsson H., 2002. Endocrine changes in late pregnancy with special emphasis on fetal well-being. Domestic Animal Endocrinology, 23, 321-328.

Leenhouwers J.I., van der Lende T., Knol E.T., 1999. Analysis of stillbirth in different lines of pig. Livestock Production Science, 57, 243-253.

Mota-Rojas D., Martinez-Burnes J., Trujillo-Ortega M.E., Alonso-Spilsbury M., Ramirez-Necoechea R., Lopez A., 2002. Effect of oxytocin treatment in sows on umbilical cord morphology, meconium staining and neonatal mortality of piglets. American Journal of Veterinary Research, 63, 1571-1574.

Peeters C., van den Tweel E., Ioroi T., Post I., Braun K., Veldhuis W., Nicolay K., Groenendaal F., van Bel F., 2002. Pharmacological interventions in the newborn piglet in the first 24 hours after hypoxia-ischemia. A hemodynamic and electrophysiological perspective. Experimental Brain Research, 147, 200-208.

Randall G.C.B., 1978. Perinatal mortality: some problems of adaptation at birth. Advances in Veterinary Sciences and Comparative Medicine, 22, 53-81.

Randall G.C.B., 1982. Changes in fetal ad maternal blood at the end of pregnancy and during parturition in the pig. Research in Veterinary Science, 32, 278-282.

Schuijt G., 1992. Aspects of obstetrical perinatology in cattle. PhD-thesis, Utrecht University, Faculty of Veterinary Medicine, Utrecht, The Netherlands.

Schuijt G. and Taverne M.A.M., 1994. The interval between birth and sternal recumbency as an objective measure of the vitality of newborn calves. Veterinary Record, 135, 111-115.

Silver M., 1992. Parturition: spontaneous or induced preterm labour and its consequences for the neonate. Animal Reproduction Science, 28, 441-449.

Szenci O., Taverne M.A.M., Bakonyi S., Erdodi A., 1988. Comparison of pre- and postnatal acid-base status of calves with perinatal mortality in a dairy herd. Veterinary Quarterly, 10, 140-144.

Szenci O., Taverne M.A.M., Takaces E., 1989. A review of 126 caesarean sections by blood gas and the acid-base status of newborn calves. Theriogenology, 32, 667-673. 
Taverne M.A.M., Naaktgeboren C., Elsaesser F., Forsling M., van der Weijden G.C., Ellendorff F., Smidt D., 1979a. Myometrial electrical activity and plasma concentrations of progesterone, estrogen and oxytocin during late pregnancy and parturition in minipigs. Biology of Reproduction, 21, 1125-1134.

Taverne M.A.M., Naaktgeboren C., van der Weijden G.C., 1979b. Myometrial activity and expulsion of fetuses. Animal Reproduction Science, 2, 117-131.

van Dijk A.J., van Rens B.T.T.M., van der Lende T., Taverne M.A.M., 2005. Factors affecting duration of the expulsive stage of parturition and piglet birth intervals in sows with uncomplicated, spontaneous farrowings. Theriogenology, 64, 1573-1590.

van Dijk A.J., van der Lende T., Taverne M.A.M., 2006. Acid-base balance of umbilical artery blood of liveborn piglets at birth and its relation with factors affecting delivery of individual piglets. Theriogenology, 66, 1824-1833.

van der Lende T. and van Rens B.T.T.M., 2003. Critical periods for foetal mortality in gilts identified by analysing the length distribution of mummified foetuses and frequency of non-fresh stillborn piglets. Animal Reproduction Science, 75, 141-150.

van Werven T., Schukken Y.H., Lloyd J., Brand A., Heeringa H.T.J., Shea M., 1992. Milk production, postpartum disease and culling rate. Theriogenology, 37, 1191-1197.

Zaleski H.M. and Hacker R.R., 1993. Variables related to the progress of parurition and probability of stillbirth in swine. Canadian Veterinary Journal, 34, 109-113. 